\title{
On Construction of Modified Class of Estimators for Population Variance using Auxiliary Attribute
}

\author{
Chandni Kumari, Ratan Kumar Thakur
}

\begin{abstract}
In this paper, an improved estimator for population variance has been proposed to improvise the log-type estimators proposed by Kumari et al. (2019). The properties of proposed estimators are derived up to the first order of approximation. The proposed estimator found to be better than the existing estimators in the sense of mean squraed error and percent relative efficiency. A numerical study is included to support the use of the suggested classes of estimators.
\end{abstract}

Keywords: Population Variance Estimators in The Sense of Mean Squared Error

\section{INTRODUCTION}

In sample surveys, auxiliary information always plays a vital role in better estimation of the parameter under investigation. This information can be used to improve the precision of the estimators. The suitable utilization of this auxiliary information can reduce the MSE of the sample mean, thus resulting in more efficient estimators. This paper, is an attempt to extend the powerful Searls approach to the traditional estimators using auxiliary information regard- ing to variables in simple random sampling. Many authors like, Singh et al. (1973), Das and Tripathi (1978), Sisodia and Dwivedi (1981), Isaki (1983), Bahl and Tuteja (1991), Prasad and Singh (1992), Swain (1994), Garcia and Cebrian (1996), Upadhaya and Singh (2001), Kalidar and Cingi (2006a, 2006b); Gupta and Shabbir (2006, 2007), Yadav and Kadilar (2013, 2014) had proposed an improved ratio estimators using Searls type estimators. Recently, Bhushan et al. (2017) among others; Kumari et al. (2019) have made the use of logarithmic relationship between the auxiliary attribute and study variable as logarithm func- tion which is very common in various branches of science as well as non-science disciplines. In this paper, some improved logarithmic estimators are proposed for improving the efficiency of the Kumari (2019) estimators as these classes of estimators are expected to improve the mean squared error. The proposed estimators would work considerably well in case when the study variable is logarithmically related to the auxiliary attribute.

Consider a finite population $U=U_{1}, U_{2}, \ldots, U_{N}$ of size $N$ from which a sample of size $\mathrm{n}$ is drawn according to simple random sampling without replacement (SRSWOR). Let $y_{i}$ and $f_{i}$ denotes the values of the study and auxiliary attribute for the ith unit $(i=1,2, \ldots, N)$, of the population. Further, let $\bar{y}_{\text {and }}$ $\bar{f}$ be the sample means and

Revised Manuscript Received on May 15, 2020.

* Correspondence Author

Chandni Kumari*, Department of Statistics, Babasaheb Bhimrao Ambedkar University, Lucknow (U.P), India.

Ratan Kumar Thakur, Department of Statistics, Babasaheb Bhimrao Ambedkar University, Lucknow (U.P), India.

(C) The Authors. Published by Blue Eyes Intelligence Engineering and Sciences Publication (BEIESP). This is an open access article under the CC BY-NC-ND license (http://creativecommons.org/licenses/by-nc-nd/4.0/)

$$
\begin{aligned}
& s_{y}^{2}=\sum_{i=1}^{n}\left(y_{i}-\bar{y}\right)^{2} /(n-1) \text { and } \\
& s_{f}^{2}=\sum_{i=1}^{n}\left(f_{i}-\bar{f}\right)^{2} /(n-1) \text { be the sample variance } \\
& \text { of the study and auxiliary attribute respectively }
\end{aligned}
$$

\section{ESTIMATORS AVAILABLE IN LITERATURE}

\subsection{Conventional Variance Estimator}

$$
t_{0}=s_{y}^{2}
$$

The bias and variance of $t_{0}$ to the first order of approfimation, are given a

$$
\begin{aligned}
& B\left(t_{0}\right)=0 \\
& V\left(t_{0}\right)=S_{y}^{4} I b_{2 y}^{*}
\end{aligned}
$$

\subsection{Isaki Ratio Estimator}

$$
t_{1}=s_{y}^{2}\left(\frac{S_{f}^{2}}{s_{f}^{2}}\right)
$$

The bias and MSE of $t_{1}$ to the first degree of approfimation are given as

$$
\begin{aligned}
& B\left(t_{1}\right)=S_{y}^{2} I\left[b_{2 f}^{*}-I_{22 y f}^{*}\right] \\
& V\left(t_{1}\right)=S_{y}^{4} I\left[b_{2 y}^{*}+b_{2 f}^{*}-2 I_{22 y f}^{*}\right]
\end{aligned}
$$

\subsection{Conventional Product Estimator}

$$
t_{2}=s_{y}^{2}\left(\frac{s_{f}^{2}}{S_{f}^{2}}\right)
$$

The bias and MSE of $t_{2}$ upto the first order of approfimation are given as

$$
\begin{aligned}
& B\left(t_{2}\right)=S_{y}^{4} I I_{22}^{*} \\
& V\left(t_{2}\right)=S_{y}^{4} I\left[b_{2 y}^{*}+b_{2 f}^{*}+2 I_{22 y f}^{*}\right]
\end{aligned}
$$

\subsection{Isaki Regression Estimator}

Isaki (1983) suggested the following regression estimator for population variance

$$
t_{3}=s_{y}^{2}+b\left(S_{f}^{2}-s_{f}^{2}\right)
$$

where $b$ is a sample regression coefficient whose population regression coefficient is $\beta$.

Published By:

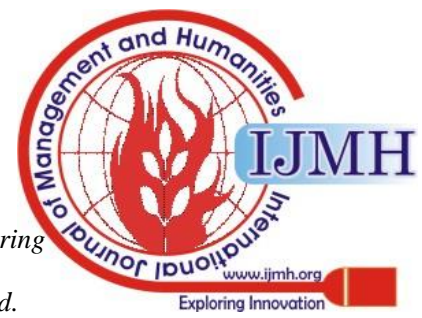


The bias and MSE of $t_{3}$ to the first degree of approfimation are given as

$$
\begin{aligned}
B\left(t_{3}\right) & =0 \\
M S E\left(t_{3}\right) & =S_{y}^{4} I\left[b_{2 y}^{*}-\frac{I_{22 y f}^{* 2}}{b_{2 f}^{*}}\right]
\end{aligned}
$$

\subsection{Singh et al. Estimator}

Singh et al. (1973) Considered the Following Estimator

$$
t_{4}=\alpha_{4} s_{y}^{2}
$$

Where $\alpha_{4}$ is a Searl (1964) constant. The optimum value of Searls constant is $\alpha_{4}=n /\left(n+b_{2 y}^{*}\right)$ for which the mean squared error is minimum.

$$
M S E\left(t_{4}\right)_{o p t}=S_{y}^{4} I\left[\frac{n b_{2 y}^{*}}{n+b_{2 y}^{*}}\right]
$$

\subsection{Das and Tripathi Estimator}

$$
t_{5}=s_{y}^{2}\left[\frac{S_{f}^{2}}{S_{f}^{2}+\alpha_{5}\left(s_{f}^{2}-S_{f}^{2}\right)}\right]
$$

Where $\alpha_{5}$ is a constant. The bias and MSE of $t_{5}$ to the first degree of approfimation is given as

$$
\begin{aligned}
B\left(t_{5}\right) & =S_{y}^{2} I\left[\alpha_{5}^{2} b_{2 y}^{*}-\alpha_{5} I_{22 y f}^{*}\right] \\
V\left(t_{5}\right) & =S_{y}^{4} I\left[b_{2 y}^{*}+\alpha_{5}^{2} b_{2 f}^{*}-2 \alpha_{5} I_{22 y f}^{*}\right]
\end{aligned}
$$

The MSE of $t_{5}$ is optimum for $\alpha_{5}=I_{22}^{*} / b_{2 f}^{*}$ and is given by

$$
M S E\left(t_{5}\right)_{o p t}=S_{y}^{4} I\left[b_{2 y}^{*}-\frac{I_{22}^{* 2}}{b_{2 f}^{*}}\right]
$$

\subsection{Prasad and Singh Estimator}

Prasad and Singh (1992) introduced the following estimator

$$
t_{6}=\alpha_{6}\left(s_{y}^{2} \frac{S_{f}^{2}}{s_{f}^{2}}\right)
$$

where $\alpha_{6}$ is a Searls content.

The bias and MSE of $t_{6}$ to the first degree of approfimation is given as

$$
\begin{aligned}
& B\left(t_{6}\right)=S_{y}^{2} I\left[\alpha_{6}\left(n+b_{2 f}^{*}-I_{22 y f}^{*}\right)-n\right] \\
& V\left(t_{6}\right)=S_{y}^{4} I\left[\alpha_{6}^{2}\left(n+b_{2 y}^{*}+3 b_{2 f}^{*}-4 I_{22 y f}^{*}\right)-2 \alpha_{6}\left(n+b_{2 f}^{*}-I_{22 y f}^{*}\right)-n\right]
\end{aligned}
$$$$
\text { The MSE of } t_{6} \text { is optimum for } \alpha_{6}=\left(n+b_{2 f}^{*}-I_{22 y f}^{*}\right) /\left(n+b_{2 y}^{*}+3 b_{2 f}^{*}-4 I_{22 y f}^{*}\right)
$$
and is given as

$$
M S E\left(t_{6}\right)_{o p t}=S_{y}^{4} I\left[n-\frac{\left(n+b_{2 f}^{*}-I_{22 y f}^{*}\right)^{2}}{n+b_{2 y}^{*}+3 b_{2 f}^{*}-4 I_{22 y f}^{*}}\right]
$$

\subsection{Garcia and Cebrian estimator}

Garcia and Cebrian (1996) introduced the following estimator

$$
t_{7}=s_{y}^{2}\left(\frac{S_{f}^{2}}{s_{f}^{2}}\right)^{\alpha_{7}}
$$

where $\alpha_{7}$ is a Searls contant.

The bias and MSE of $t_{7}$ to the first degree of approfimation is given as

$$
\begin{aligned}
B\left(t_{7}\right) & =S_{y}^{2} I\left[\alpha_{7} \frac{\left(\alpha_{5}+1\right)}{2} b_{2 f}^{*}-\alpha_{7} I_{22 y f}^{*}\right] \\
V\left(t_{7}\right) & =S_{y}^{4} I\left[b_{2 y}^{*}+\alpha_{7}^{2} b_{2 f}^{*}-2 \alpha_{7} I_{22 y f}^{*}\right]
\end{aligned}
$$

The MSE of $t_{7}$ is optimum for $\alpha_{7}=I_{22}^{*} / b_{2 f}^{*}$ and is given as

$$
M S E\left(t_{7}\right)_{o p t}=S_{y}^{4} I\left[b_{2 y}^{*}-\frac{I_{22 y f}^{* 2}}{b_{2 f}^{*}}\right]
$$

\subsection{Upadhaya and Singh Estimator}

Upadhaya and Singh (2001) suggested following estimator

$$
t_{8}=s_{y}^{2}+\alpha_{6}\left(S_{f}^{2}-s_{f}^{2}\right)
$$

where $\alpha_{8}$ is a constant. The MSE of $t_{8}$ to the first degree of approfimation is given as

$$
V\left(t_{8}\right)=S_{y}^{4} I\left[b_{2 y}^{*}+\alpha_{8}^{2} \frac{S_{f}^{4}}{S_{y}^{4}} b_{2 f}^{*}-2 \alpha_{8} \frac{S_{f}^{2}}{S_{y}^{2}} I_{22 y f}^{*}\right]
$$

The MSE of $t_{8}$ is optimum for $\alpha_{8}=\frac{S_{y}^{2} I_{22 y f}^{*}}{S_{f}^{2} b_{2 f}^{*}}$ and is given as

$$
M S E\left(t_{8}\right)_{o p t}=S_{y}^{4} I\left[b_{2 y}^{*}-\frac{I_{22 y f}^{* 2}}{b_{2 f}^{*}}\right]
$$

\subsection{Shabbir and Gupta (2006) estimator}

Sabbir and Gupta (2006) Proposed the Following Estimator

$$
t_{9}=\lambda t_{m}
$$

where $\lambda$ is a Searls (1964) contant whose value is to be determined later. Here $t_{m}$ is a combination of Singh et al. (1973), Prasad and Singh (1992) and is defined as

Published By:

Blue Eyes Intelligence Engineering 10

\& Sciences Publication

(C) Copyright: All rights reserved.

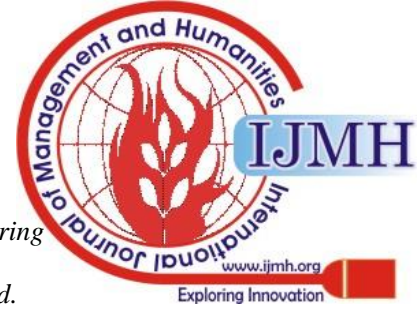




$$
t_{m}=K_{1} s_{y}^{2}+K_{2} s_{y}^{2}\left(\frac{S_{f}^{2}}{s_{f}^{2}}\right)
$$

where $K_{1}$ and $K_{2}$ are the weights such that $K_{1}+K_{2}=1$ The bias and MSE of $t_{9}$ to the first degree of approfimation is given as

$$
\begin{aligned}
& B\left(t_{9}\right)=S_{y}^{2} I\left[\lambda\left\{n+K_{2}\left(b_{2 f}^{*}-I_{22 y f}^{*}\right)\right\}-n\right] \\
& V\left(t_{9}\right)=S_{y}^{4} I\left[\lambda^{2}\left(n+b_{2 y}^{*}+K_{2}^{2} b_{2 f}^{*}+2 K_{2} b_{2 f}^{*}-4 K_{2} I_{22 y f}^{*}\right)-2 \lambda\left(n+K_{2} b_{2 f}^{*}-K_{2} I_{22 y f}^{*}\right)+n\right]
\end{aligned}
$$

The MSE of $t_{9}$ is optimum for

$$
\begin{gathered}
\lambda=\frac{n+K_{2}\left(b_{2 f}^{*}-I_{22 y f}^{*}\right)}{n+b_{2 y}^{*}+K_{2}^{2} b_{2 f}^{*}+2 K_{2} b_{2 f}^{*}-4 K_{2} I_{22 y f}^{*}} \text { and } \\
K_{2}=\frac{I_{22}^{*}}{b_{2 f}^{*}} \text { is given as } \\
M S E\left(t_{9}\right)_{\text {opt }}=S_{y}^{4} I\left[n-\frac{\left\{n+I_{22 y f}^{*}-\frac{I_{22 y f}^{* 2}}{b_{2 f}^{*}}\right\}^{2}}{n+b_{2 y}^{*}+2 I_{22 y f}^{*}-3 \frac{I_{22 y f}^{* 2}}{b_{2 f}^{*}}}\right]
\end{gathered}
$$

\subsection{Shabbir and Gupta (2007) estimator}

$$
t_{10}=k_{1} s_{y}^{2}+k_{2}\left(S_{f}^{2}-s_{f}^{2}\right) \operatorname{efp}\left(\frac{S_{f}^{2}-s_{f}^{2}}{S_{f}^{2}+s_{f}^{2}}\right)
$$

where $k_{1}$ and $k_{2}$ are suitably chosen coantants.

\section{Situation 1. $k_{1}+k_{2}=1$}

The bias and MSE of $t_{10}$ the first degree of approfimation are given as

$$
\begin{aligned}
B\left(t_{10}\right)= & \left(k_{1}-1\right)\left(S_{y}^{2}-\frac{1}{2} S_{f}^{2} I b_{2 f}^{*}\right)+k_{1} S_{y}^{2} I\left[\frac{3}{8} b_{2 f}^{*}-\frac{1}{2} I_{22 y f}^{*}\right] \\
V\left(t_{10}\right)= & S_{y}^{4} I\left[k_{1}^{2}\left\{n+b_{2 y}^{*}+\frac{S_{f}^{4}}{S_{y}^{4}} b_{2 f}^{*}+\frac{1}{4} b_{2 f}^{*}-I_{22 y f}^{*}+2 \frac{S_{f}^{2}}{S_{y}^{2}}\left(I_{22 y f}^{*}-\frac{1}{2} b_{2 f}^{*}\right)\right\}\right] \\
& -2 S_{y}^{4} I k_{1}\left[\left\{n+\frac{S_{f}^{4}}{S_{y}^{4}}+\frac{S_{f}^{2}}{S_{y}^{2}}\left(I_{22 y f}^{*}-\frac{1}{2} b_{2 f}^{*}\right) b_{2 f}^{*}-K_{2} I_{22 y f}^{*}\right\}+n+\frac{S_{f}^{4}}{S_{y}^{4}} b_{2 f}^{*}\right]
\end{aligned}
$$

The optimum value of $k_{1}$ which minimizes $\operatorname{MSE}\left(t_{10}\right)$ is given as

$$
k_{1}=\frac{A_{1}+A_{3}}{A_{1}+A_{2}+2 A_{3}} \text {, where } A_{1}=n+\frac{S_{f}^{4}}{S_{y}^{4}} b_{2 f}^{*}, A_{2}=b_{2 y}^{*}+\frac{1}{4} b_{2 f}^{*}-I_{22 y f}^{*}, A_{3}=\frac{S_{f}^{2}}{S_{y}^{2}}\left(I_{22 y f}^{*}-\frac{1}{2} b_{2 f}^{*}\right)
$$




$$
M S E\left(t_{10}\right)_{o p t}=S_{y}^{4} I\left[A_{1}-\frac{\left(A_{1}+A_{3}\right)^{2}}{A_{1}+A_{2}+2 A_{3}}\right]
$$

Situation 2. Unconstrained choice of $k_{1}$ and $k_{2}$

The bias and MSE of $t_{10}^{*}$ the first degree of approfimation are given as

$$
\begin{aligned}
& B\left(t_{10}^{*}\right)=\left(k_{1}-1\right) S_{y}^{2}+\frac{1}{2} K_{2} S_{f}^{2} I b_{2 f}^{*}+k_{1} S_{y}^{2} I\left[\frac{3}{8} b_{2 f}^{*}-\frac{1}{2} I_{22 y f}^{*}\right] \\
& M S E\left(t_{10}^{*}\right)_{\text {opt }}=S_{y}^{4} I\left[\frac{\operatorname{Var}\left(\hat{S}_{\text {reg }}\right)}{\left.1+\frac{\operatorname{Var}\left(\hat{S}_{\text {reg }}\right)}{S_{y}^{4}}\right]}\right]
\end{aligned}
$$

\subsection{Kalidar and Cingi estimator}

Kadilar and Cingi (2006) suggested the following ratio type estimator

$$
\begin{aligned}
& t_{11}=\omega_{1} s_{y}^{2}+\omega_{2}\left(s_{y}^{2} \frac{S_{f}^{2}}{s_{f}^{2}}\right) \tau \\
& \text { where } \omega_{1}+\omega_{2}=1
\end{aligned}
$$

The optimum bias and MSE of $t_{11}$ are given as

$$
\begin{aligned}
& B\left(t_{11}\right)=S_{y}^{2}\left[\left(\omega_{1}^{*}-1\right)+\omega_{1}^{*} \tau I b_{2 f}^{*}\right] \\
& M S E\left(t_{11}\right)=S_{y}^{4} I\left[\lambda^{2}\left(n+b_{2 y}^{*}+K_{2}^{2} b_{2 f}^{*}+2 K_{2} b_{2 f}^{*}-4 K_{2} I_{22 y f}^{*}\right)-2 \lambda\left(n+K_{2} b_{2 f}^{*}-K_{2} I_{22 y f}^{*}\right)+n\right] \\
& \text { The MSE of } t_{11} \text { is optimum for } \lambda=\frac{n+K_{2}\left(b_{2 f}^{*}-I_{22 y f}^{*}\right)}{n+b_{2 y}^{*}+K_{2}^{2} b_{2 f}^{*}+2 K_{2} b_{2 f}^{*}-4 K_{2} I_{22 y f}^{*}} \text { and } \\
& K_{2}=\frac{I_{22 y f}^{*}}{b_{2 f}^{*}} \text { is given by } \\
& M S E\left(t_{11}\right)_{o p t}=S_{y}^{4} I\left[n-\frac{\left\{n+I_{22 y f}^{*}-\frac{I_{22}^{* 2}}{b_{2 f}^{*}}\right\}^{2}}{n+b_{2 y}^{*}+2 I_{22 y f}^{*}-3 \frac{I_{22}^{* 2}}{b_{2 f}^{*}}}\right]
\end{aligned}
$$

2.13. Yadav and Kalidar (2013) Estimator

Yadav and Kadilar (2013) introduced the following estimator

$$
t_{12}=s_{y}^{2} \operatorname{efp}\left[1-\frac{a_{12} s_{f}^{2}}{S_{f}^{2}+\left(a_{12}-1\right) s_{f}^{2}}\right]
$$

where $a_{12}$ is a constant. The optimum MSE of $t_{12}$ to the first degree of approfimation is given as 


$$
\operatorname{MSE}\left(t_{12}\right)_{o p t}=S_{y}^{4} I\left\{b_{2 y}^{*}-\frac{I_{22 y f}^{* 2}}{b_{2 f}^{*}}\right\}
$$

\subsection{Yadav and Kadilar (2014) estimator}

Yadav and Kadilar (2014) introduced the following ratio-product-ratio estimator

$$
t_{\alpha, \beta_{12}}=\alpha\left[\frac{(1-\beta) s_{f}^{2}+\beta S_{f}^{2}}{\beta s_{f}^{2}+(1-\beta) S_{f}^{2}}\right] s_{y}^{2}+(1-\alpha)\left[\frac{\beta s_{f}^{2}+(1-\beta) S_{f}^{2}}{(1-\beta) s_{f}^{2}+\beta S_{f}^{2}}\right] s_{y} 2
$$

where $a$ and $\beta$ are real constant. The optimum MSE of $t_{a, \beta_{12}}$ to the first degree of approfima-tion is given as

$$
M S E\left(t_{12}^{*}\right)_{\text {opt }}=S_{y}^{4} I b_{2 y}^{*}
$$

\section{THE PROPOSED ESTIMATORS}

We propose the following new classes of log-type estimators for the population variance $S_{y}^{2}$ as:

$$
\begin{aligned}
& T_{1}^{*}=w_{1} s_{y}^{2}\left[1+\log \left(\frac{S_{f}^{2}}{s_{f}^{2}}\right)\right]^{a_{1}} \\
& T_{2}^{*}=w_{2} s_{y}^{2}\left[1+a_{2} \log \left(\frac{S_{f}^{2}}{s_{f}^{2}}\right)\right] \\
& T_{3}^{*}=w_{3} s_{y}^{2}\left[1+\log \left(\frac{S_{f}^{* 2}}{s_{f}^{* 2}}\right)\right]^{a_{3}} \\
& T_{4}^{*}=w_{4} s_{y}^{2}\left[1+a_{4} \log \left(\frac{S_{f}^{* 2}}{s_{f}^{* 2}}\right)\right]
\end{aligned}
$$

where

$$
\begin{aligned}
& s_{f}^{* 2}=a s_{f}^{2}+b \\
& S_{f}^{* 2}=a S_{f}^{2}+b
\end{aligned}
$$

such that $a(\neq 0), b$ are either real numbers or functions of the known parameters of the auxiliary attribute $f$ such as the standard deviations $S_{f}$, coefficient of variation $C_{f}$, coefficient of kurtosis $b_{2 f}$, coefficient of skewness $b_{1 f}$, and correlation coefficient $\rho$ of the population. If $a_{i}=0$, then the proposed estimator becomes the usual per unit

\section{BIAS AND MSE OF PROPOSED ESTIMATORS}

In this paper, the biases and the mean square error(s) of all the estimators are considered up to the terms of order $n^{-1}$.

Theorem 1. The bias and MSE of $T_{1}^{*}$ are given by

$$
\begin{aligned}
\operatorname{Bias}\left(T_{1}^{*}\right) & =S_{y}^{2}\left[w_{1}\left\{1-a_{1} I I_{22 y f}^{*}+a_{1} I b_{2 f}^{*}+\frac{a_{1}^{2}}{2} I b_{2 f}^{*}\right\}-1\right] \\
\operatorname{MSE}\left(T_{1}^{*}\right) & =S_{y}^{4} w_{1}^{2}\left[1+I\left(b_{2 y}^{*}+2 a_{1}^{2} b_{2 f}^{*}-4 a_{1} b_{2 f}^{*}+2 a_{1} b_{2 f}^{*}\right)\right] \\
& -2 S_{y}^{4} w_{1}\left[1+I\left(a_{1} b_{2 f}^{*}+\frac{a_{1}^{2} b_{2 f}^{*}}{2}-a_{1} I_{22}^{*}\right)\right]+1
\end{aligned}
$$

Proof. Consider the estimator, 


$$
\begin{aligned}
T_{1}^{*} & =w_{1} s_{y}^{2}\left[1+\log \left(\frac{S_{f}^{2}}{s_{f}^{2}}\right)\right]^{a_{1}} \\
& =w_{1} S_{y}^{2}\left(1+\epsilon_{0}\right)\left[1-\log \left(1+\epsilon_{1}\right)\right]^{a_{1}} \\
& =w_{1} S_{y}^{2}\left(1+\epsilon_{0}\right)\left[1-a_{1}\left(\epsilon_{1}-\frac{\epsilon_{1}^{2}}{2}\right)+\left(\frac{a_{1}\left(a_{1}+1\right)}{2}\right)\left(\epsilon_{1}-\frac{\epsilon_{1}^{2}}{2}\right)^{2}\right] \\
& =\left(w_{1}-1\right) S_{y}^{2}+w_{1} S_{y}^{2}\left[\epsilon_{0}-a_{1}\left(\epsilon_{1}-\frac{\epsilon_{1}^{2}}{2}\right)+\frac{a_{1}\left(a_{1}+1\right)}{2} \epsilon_{1}^{2}-a_{1} \epsilon_{0} \epsilon_{1}\right]
\end{aligned}
$$

Squaring on both the sides and then taking expectation over the proposed estimator, we have

$$
\begin{aligned}
& =S_{y}^{4}\left(w_{1}-1\right)^{2}+w_{1}^{2} S_{y}^{4}\left[E\left(\epsilon_{0}\right)^{2}+a_{1}^{2} E\left(\epsilon_{1}\right)^{2}+2 a_{1} E\left(\epsilon_{0} \epsilon_{1}\right)\right] \\
& +2 S_{y}^{4} \quad w_{1}\left(w_{1}-1\right)\left[a_{1} E\left(\epsilon_{1}\right)^{2}-a_{1} E\left(\epsilon_{0} \epsilon_{1}\right)+\frac{a_{1}^{2}}{2} E\left(\epsilon_{1}^{2}\right)\right]
\end{aligned}
$$

Using the results from Sukhatme and Sukhatme, we have

$E\left(\epsilon_{0}\right)=0=E\left(\epsilon_{1}\right), E\left(\epsilon_{0}\right)^{2}=I b_{2 y}^{*}, E\left(\epsilon_{1}\right)^{2}=I b_{2 f}^{*}, E\left(\epsilon_{0} \epsilon_{1}\right)=I I_{22}^{*}$ where $b_{2 f}^{*}=b_{2 f}-1$, $b_{2 y}^{*}=b_{2 y}-1$ and $I_{22}^{*}=I_{22}-1 ; I_{p q}=m_{p q} / m_{20}^{\frac{p}{2}} m_{02}^{\frac{q}{2}}, \quad m_{p q}=\sum_{i=1}^{N}\left(Y_{i}-\bar{Y}\right)^{p}\left(Y_{i}-\bar{Y}\right)^{q} / N, I=1 / N$, $b_{2 y}=m_{40} / m_{20}^{2}, \quad b_{2 f}=m_{04} / m_{02}^{2}$ are the coefficient of kurtosis of $y$ and $f$ respectively.

Now, substituting the above results in (4:1), we get,

$$
\begin{aligned}
\operatorname{MSE}\left(T_{1}^{*}\right) & =S_{y}^{4} w_{1}^{2}\left[1+I\left(b_{2 y}^{*}+2 a_{1}^{2} b_{2 f}^{*}-4 a_{1} I_{22 y f}^{*}+2 a_{1} b_{2 f}^{*}\right]\right. \\
& -2 S_{y}^{4} w_{1}\left[1+I\left(a_{1} b_{2 f}^{*}+\frac{a_{1}^{2} b_{2 f}^{*}}{2}-a_{1} I_{22 y f}^{*}\right)\right]+1
\end{aligned}
$$

Corollary 2. The optimum value of $a_{1}$ and $w_{1}$ are

where

$$
\begin{aligned}
& a_{1}=\frac{I_{22}^{*}}{b_{2 f}} \\
& w_{1}=\frac{B_{1}}{A_{1}}
\end{aligned}
$$

$$
\begin{aligned}
& A_{1}=1+I\left(b_{2 y}^{*}+2 a_{1}^{2} b_{2 f}^{*}-4 a_{1} I_{22 y f}^{*}+2 a_{1} b_{2 f}^{*}\right. \\
& B_{1}=1+I\left(a_{1} b_{2 f}^{*}+\frac{a_{1}^{2} b_{2 f}^{*}}{2}-a_{1} I_{22 y f}^{*}\right)
\end{aligned}
$$

respectively. Also, the minimum mean squared error of $T_{1}^{*}$ is

$$
M S E\left(T_{1}^{*}\right)_{\text {opt }}=S_{y}^{4} I\left[1-\frac{B_{1}^{2}}{A_{1}}\right]
$$

Proof. Obvious, using Theorem 1.

Theorem 3. The bias and MSE of $T_{2}^{*}$ are given by 


$$
\begin{aligned}
\operatorname{Bias}\left(T_{2}^{*}\right)= & S_{y}^{2}\left[w_{2}\left\{1-a_{2} I I_{22 y f}^{*}+\frac{a_{2}^{2}}{2} I b_{2 f}^{*}\right\}-1\right] \\
\operatorname{MSE}\left(T_{2}^{*}\right)= & S_{y}^{4} w_{2}^{2}\left[1+I\left(b_{2 y}^{*}+a_{2}^{2} b_{2 f}^{*}-4 a_{2} I_{22 y f}^{*}+a_{2} b_{2 f}^{*}\right]\right. \\
& -2 S_{y}^{4} w_{2}\left[1+I\left(\frac{a_{2} b_{2 f}^{*}}{2}-a_{2} I_{22 y f}^{*}\right)\right]+1
\end{aligned}
$$

respectively.

Proof. Trivial and similar to derivation of Theorem 1, hence omitted.

Corollary 4. The optimum value of $a_{2}$ and $w_{2}$ are

$$
a_{2}=\frac{I_{22}^{*}}{b_{2 f}^{*}} \quad \text { and } \quad w_{2}=\frac{B_{2}}{A_{2}}
$$

where

$$
\begin{aligned}
& A_{2}=1+I\left(b_{2 y}^{*}+a_{2}^{2} b_{2 f}^{*}-4 a_{2} I_{22 y f}^{*}+a_{2} b_{2 f}^{*}\right) \\
& B_{2}=1+I\left(\frac{a_{2} b_{2 f}^{*}}{2}-a_{2} I_{22 y f}^{*}\right)
\end{aligned}
$$

Also, the minimum mean squared error of $T_{2}^{*}$ is

$$
M S E\left(T_{2}^{*}\right)_{o p t}=S_{y}^{4}\left[1-\frac{B_{2}^{2}}{A_{2}}\right]
$$

Theorem 5. The bias and MSE of $T_{3}^{*}$ are given by

$$
\begin{aligned}
\operatorname{Bias}\left(T_{3}^{*}\right)= & S_{y}^{2}\left[w_{1}\left\{1-a_{3} \eta I I_{22 y f}^{*}+a_{3} \eta^{2} I b_{2 f}^{*}+\frac{a_{3}^{2}}{2} I \eta^{2} b_{2 f}^{*}\right\}-1\right] \\
\operatorname{MSE}\left(T_{3}^{*}\right)= & S_{y}^{4} w_{1}^{2}\left[1+I\left(b_{2 y}^{*}+2 a_{3}^{2} \eta^{2} b_{2 f}^{*}-4 a_{3} \eta b_{2 f}^{*}+2 a_{3} \eta^{2} b_{2 f}^{*}\right)\right] \\
& -2 S_{y}^{4} w_{1}\left[1+I\left(a_{3} \eta^{2} b_{2 f}^{*}+\frac{a_{3}^{2}}{2} \eta^{2} b_{2 f}^{*}-a_{3} \eta I_{22 y f}^{*}\right)\right]+1
\end{aligned}
$$

respectively.

Proof. Trivial and similar to derivation of Theorem 1, hence omitted.

Corollary 6. The optimum value of $a_{3}$ and $w_{3}$ is

$$
a_{3}=\frac{I_{22 y f}^{*}}{b_{2 f}^{*}} \quad \text { and } \quad w_{3}=\frac{B_{3}}{A_{3}}
$$

where

$$
\begin{aligned}
& A_{3}=1+I\left(b_{2 y}^{*}+2 a_{3}^{2} \eta^{2} b_{2 f}^{*}-4 a_{3} \eta b_{2 f}^{*}+2 a_{3} \eta^{2} b_{2 f}^{*}\right) \\
& B_{3}=1+I\left(a_{3} \eta^{2} b_{2 f}^{*}+\frac{a_{3}^{2}}{7} \eta^{2} b_{2 f}^{*}-a_{3} \eta I_{22 y f}^{*}\right)
\end{aligned}
$$

Also, the minimum mean squared error of $T_{3}^{*}$ is 


$$
M S E\left(T_{3}^{*}\right)_{o p t}=S_{y}^{4}\left[1-\frac{B_{3}^{2}}{A_{3}}\right]
$$

Theorem 7. The bias and MSE of $T_{4}^{*}$ are given by

respectively.

$$
\begin{aligned}
\operatorname{Bias}\left(T_{4}^{*}\right)= & S_{y}^{2}\left[w_{4}\left\{1-a_{4} \eta I I_{22 y f}^{*}+\frac{a_{4}^{2}}{2} I \eta^{2} b_{2 f}^{*}\right\}-1\right] \\
\operatorname{MSE}\left(T_{4}^{*}\right)= & S_{y}^{4} w_{4}^{2}\left[1+I\left(b_{2 y}^{*}+a_{4}^{2} \eta^{2} b_{2 f}^{*}-4 a_{4} \eta I_{22 y f}^{*}+a_{4} \eta^{2} b_{2 f}^{*}\right]\right. \\
& -2 S_{y}^{4} w_{2}\left[1+I\left(\frac{a_{4} \eta^{2} b_{2 f}^{*}}{2}-a_{4} \eta I_{22 y f}^{*}\right)\right]+1
\end{aligned}
$$

Proof. Trivial and similar to derivation of Theorem 1, hence omitted.

Corollary 8. The optimum value of $a_{4}$ and $w_{4}$ is

$$
a_{4}=\frac{I_{22}^{*}}{b_{2 f}^{*}} \quad \text { and } \quad w_{4}=\frac{B_{4}}{A_{4}}
$$

where

$$
\begin{aligned}
& A_{4}=1+I\left(b_{2 y}^{*}+a_{4}^{2} \eta^{2} b_{2 f}^{*}-4 a_{4} \eta I_{22 y f}^{*}+a_{4} \eta^{2} b_{2 f}^{*}\right. \\
& B_{4}=1+I\left(\frac{a_{4} \eta^{2} b_{2 f}^{*}}{2}-a_{4} \eta I_{22 y f}^{*}\right)+1
\end{aligned}
$$

Also, the minimum mean squared error of $T_{3}^{*}$ is

$$
\operatorname{MSE}\left(T_{4}^{*}\right)_{o p t}=S_{y}^{4}\left[1-\frac{B_{4}^{2}}{A_{4}}\right]
$$

\section{COMPARISON OF ESTIMATORS}

In this section, we compare the proposed classes of estimators with some important estimators. The comparison will be in terms of their MSEs up to the order of $n^{-1}$ Let us define

$$
\begin{aligned}
& C_{1}=b_{2 y}^{*}+b_{2 f}^{*}-2 I_{22}^{*}, C_{2}=b_{2 y}^{*}+b_{2 f}^{*}-2 I_{22}^{*}, D=b_{2 y}^{*} b_{2 f}^{*}-I_{22}^{*}, E=\left[\frac{n b_{2 y}^{*}}{n+b_{2 y}^{*}}\right], F= \\
& {\left[n-\frac{\left(n+b_{2 f}^{*}-I_{22}^{*}\right)^{2}}{n+b_{2 y}^{*}+3 b_{2 f}^{*}-4 I_{22}^{*}}\right], G=\left[n-\frac{\left.\left\{n+I_{22}^{*}-\frac{I_{22}^{* 2}}{b_{2 f}^{*}}\right\}^{2}\right]}{n+b_{2 y}^{*}+2 I_{22}^{*}-3 \frac{I_{22}^{* 2}}{b_{2 f}^{*}}}\right], H=\left[A_{1}-\frac{\left(A_{1}+A_{3}\right)^{2}}{A_{1}+A_{2}+2 A_{3}}\right] .}
\end{aligned}
$$




$$
\begin{aligned}
& M S E\left(t_{0}\right)>M S E\left(T_{1}^{*}\right)_{o p t} \text { if } \quad b_{2 y}^{*}+\frac{B^{2}}{A}-n>0 \\
& M S E\left(t_{1}\right)>M S E\left(T_{1}^{*}\right)_{o p t} \text { if } C_{1}-n+\frac{B^{2}}{A}>0 \\
& M S E\left(t_{2}\right)>M S E\left(T_{1}^{*}\right)_{o p t} \text { if } C_{2}-n+\frac{B^{2}}{A}>0 \\
& M S E\left(t_{3}\right)>M S E\left(T_{1}^{*}\right)_{o p t} \text { if } D-\left(n-\frac{B^{2}}{A}\right) b_{2 f}^{*}>0 \\
& M S E\left(t_{4}\right)>M S E\left(T_{1}^{*}\right)_{o p t} \text { if } E-n-\frac{B^{2}}{A}>0 \\
& M S E\left(t_{5}\right)>M S E\left(T_{1}^{*}\right)_{o p t} \text { if } D-\left(n-\frac{B^{2}}{A}\right) b_{2 f}^{*}>0 \\
& M S E\left(t_{6}\right)>M S E\left(T_{1}^{*}\right)_{o p t} \text { if } \quad F-n+\frac{B^{2}}{A}>0 \\
& M S E\left(t_{7}\right)>M S E\left(T_{1}^{*}\right)_{o p t} \text { if } \quad D-\left(n-\frac{B^{2}}{A}\right) b_{2 f}^{*}>0 \\
& M S E\left(t_{8}\right)>M S E\left(T_{1}^{*}\right)_{o p t} \text { if } D-\left(n-\frac{B^{2}}{A}\right) b_{2 f}^{*}>0 \\
& M S E\left(t_{9}\right)>M S E\left(T_{1}^{*}\right)_{o p t} \text { if } \quad G-n+\frac{B^{2}}{A}>0 \\
& M S E\left(t_{10}\right)>M S E\left(T_{1}^{*}\right)_{o p t} \text { if } \quad H-n+\frac{B^{2}}{A}>0 \\
& M S E\left(t_{11}\right)>M S E\left(T_{1}^{*}\right)_{o p t} \text { if } \quad G-n+\frac{B^{2}}{A}>0 \\
& M S E\left(t_{12}\right)>M S E\left(T_{1}^{*}\right)_{o p t} \text { if } D-\left(n-\frac{B^{2}}{A}\right) b_{2 f}^{*}>0
\end{aligned}
$$

\section{EMPIRICAL STUDY}

To compare the efficiency of the suggested class of estimator numerically, we considered nine natural data sets. The description of the population is given below.

Population 1. (Cochran (1977), Pg. no. 107)

$y$ : number of persons per block

$f$ : number of rooms per block

$S_{y}^{2}=214.69, S_{f}^{2}=56.76, b_{2 y}^{*}=1.2387, b_{2 f}^{*}=1.3523, I_{22}^{*}=0.5432, C_{f}=0.1450, \bar{f}=58.8, \rho$

$=0.6515, n=10$.

Population 2. (Cochran (1977), Pg. no. 203)

$y$ : actual weight of peaches on each tree

$f$ : eye estimate of weight of peaches on each tree.

$S_{y}^{2}=99.81, S_{f}^{2}=85.09, b_{2 y}^{*}=0.9249, b_{2 f}^{*}=1.2932, I_{22}^{*}=1.1149, C_{f}=0.1621, \bar{f}=56.9, \rho=$ 0.9937, $n=10$. 
Population 3. (Sukhatme P. V. (1970), Pg. no. 185)

$y$ : wheat acreage in 1937

$f$ : wheat acreage in 1936

$S_{y}^{2}=26456.99, S_{f}^{2}=22355.76, b_{2 y}^{*}=2.1842, b_{2 f}^{*}=1.2030, I_{22}^{*}=1.5597, C_{f}=0.5625, \bar{f}=$ 265.8, $\rho=0.977, n=10$.

Population 4. (Singh D and Chaudhary F. S., Pg. no. 107). $y$ : number of boats landing at a particular centre

$f$ : catch of fish in quintals.

$S_{y}^{2}=201324.4, S_{f}^{2}=396.8889, b_{2 y}^{*}=0.9462, b_{2 f}^{*}=0.6078, I_{22}^{*}=0.6333, C_{f}=0.7288, \bar{X}=$ $27.3333, \rho=0.9308, n=4$.

Population 5. (Singh D and Chaudhary F. S., Pg. no. 141).

$y$ :number of bearing lime trees

$f$ :area under lime (in acres)

$S_{y}^{2}=6564586.45, S_{f}^{2}=1092.1024, b_{2 y}^{*}=12.2574, b_{2 f}^{*}=4.5788, I_{22}^{*}=6.7126, C_{f}=1.4273, \bar{X}$ $=22.6209, \rho=0.9021, n=9$.

Population 6. (Choudhary F. S. and Singh D., Pg. no. 176).

$y$ : number of cows in milk enumerated

$f$ : number of cows in milk in the previous year.

$S_{y}^{2}=332721.2079, S_{f}^{2}=281472.7868, b_{2 y}^{*}=6.2079, b_{2 f}^{*}=5.0043, I_{22}^{*}=4.9528, C_{f}=0.8276, \bar{X}=$ $641.05, \rho=0.8933, n=8$.

Population 7. (Singh S., Pg. no. 324-325). y : approximate duration of sleep (in minutes)

$f$ : age in years of the persons.

$S_{y}^{2}=3582.579, S_{f}^{2}=85.2367, b_{2 y}^{*}=1.6678, b_{2 f}^{*}=1.2389, I_{22}^{*}=0.9961, C_{f}=0.1349, \bar{X}=67.2667, \rho$ $=-0.8552, n=9$.

Population 8. (Singh S., Pg. no. 1114). y : approfimate duration of sleep (in minutes)

$f$ : age in years of the persons.

$S_{y}^{2}=0.0073, S_{f}^{2}=0.0063, b_{2 y}^{*}=2.6323, b_{2 f}^{*}=2.4016, I_{22}^{*}=1.8351, C_{f}=1.2352, \bar{X}=0.1831, \rho=0.7789$,

$n=11$.

By using the above data set, the percent relative efficiency of the different estimator are given in Table 2.

In the above table, the relative efficiency of the proposed estimator is much better as compared to other estimators for all the data sets given here.

Table 1: PRE of the Estimators with Respect to $t_{0}$

\begin{tabular}{|c|c|c|c|c|c|c|c|c|}
\hline Est. & Pop 1 & Pop 2 & Pop 3 & Pop 4 & Pop 5 & Pop 6 & Pop 7 & Pop 8 \\
\hline$t_{0}$ & 100 & 100 & 100 & 100 & 100 & 100 & 100 & 100 \\
$t_{1}$ & 121.38 & 320.81 & 815.60 & 329.16 & 359.35 & 475.29 & 182.39 & 193.03 \\
$t_{2}$ & 33.68 & 19.48 & 33.56 & 33.54 & 40.50 & 29.39 & 34.04 & 30.24 \\
$t_{3}$ & 121.38 & 639.14 & 1347.98 & 330.39 & 507.23 & 475.29 & 192.40 & 214.00 \\
$t_{4}$ & 112.38 & 639.14 & 121.42 & 30.91 & 236.19 & 177.59 & 118.53 & 123.93 \\
$t_{5}$ & 121.38 & 109.23 & 1347.98 & 330.96 & 507.23 & 475.29 & 192.40 & 214.00 \\
$t_{6}$ & 112.95 & 639.14 & 818.13 & 348.83 & 381.77 & 558.96 & 212.28 & 242.02 \\
$t_{7}$ & 121.38 & 639.14 & 1347.98 & 330.39 & 507.23 & 475.29 & 192.40 & 214.00 \\
$t_{8}$ & 121.38 & 639.14 & 1347.98 & 330.39 & 507.23 & 475.29 & 192.40 & 214.03 \\
$t_{9}$ & 143.14 & 749.18 & 1434.48 & 349.88 & 528.70 & 559.08 & 220.35 & 258.35 \\
$t_{10}$ & 121.38 & 489.41 & 306.84 & 227.25 & 319.46 & 457.77 & 188.82 & 228.88 \\
$t_{10}^{*}$ & 82.34 & 648.39 & 1369.81 & 354.05 & 643.42 & 552.89 & 210.93 & 237.93 \\
$t_{11}$ & 82.53 & 322.84 & 815.55 & 329.05 & 358.77 & 269.13 & 182.54 & 102.02 \\
$t_{12}$ & 121.38 & 639.14 & 1347.98 & 330.39 & 507.23 & 475.29 & 192.40 & 214.00 \\
$t_{\alpha, \beta_{12}}$ & 100 & 100 & 100 & 100 & 100 & 100 & 100 & 100 \\
$t_{13}$ & 121.38 & 639.14 & 1347.98 & 330.39 & 507.23 & 475.29 & 192.40 & 214.00 \\
$T_{1}^{*}$ & 147.02 & 1163.41 & 1863.95 & 439.47 & 991.93 & 2120.43 & 247.66 & 311.67 \\
\hline
\end{tabular}




\section{CONCLUSION}

The present study extends the idea of Bhushan and Kumari (2019) regarding the effective use of auxiliary information if the relationship between the study variable and the auxiliary attribute is of logarithmic type. Further, the efficiency of the proposed estimators are compared with some conventional estimators and some recent estimators of Singh et al. (1973), Das and Tripathi (1978), Sisodia and Dwivedi (1981), Isaki (1983), Bahl and Tuteja (1991), Prasad and Singh (1992), Swain (1994), Garcia and Cebrian (1996), Upadhaya and Singh (2001), Kalidar and Cingi (2006a, 2006b); Gupta and Shabbir (2006, 2007), Yadav and Kadilar (2013, 2014). The proposed estimator is most efficient than all the estimators. This study is also supported through an empirical study and the result of this study is quite encouraging.

\section{REFERENCES}

1. Kumari, C. and Thakur, R. K. (2019). Optimal Two Parameter Logarithmic Estimators for Estimating the Population Variance, Glo Jour Pure App. Math., 15 (5), 527-536.

2. Bhushan S. and Kumari C. (2019). Double Sampling Log Type Estimators Using Auxiliary Attribute for Population Variance, J. Stat. Appl. Pro., 6 (3), 1-6.

3. Bhushan S. and Kumari C. (2018). A new log type estimator for estimating the population variance, Int. J. Comp. App. Math., 13 (1), 43-54.

4. Bhushan S. and Kumari C. (2018). A Class of Double Sampling Log Type Estimators for Population Variance Using Two Auxiliary Variable, Int. J. Appl. Eng. Res., 13 (13), 11151-11155.

5. Bhushan S. and Kumari C. (2018). Estimation of Variance of Finite Population Using Double Sampling Scheme, Int. J. Sci. Eng. Res., 9 (8), 1893-1901.

6. Bhushan S. and Kumari C. (2018). Modied Ratio Estimators Using Two Auxiliary Infor- mation for Estimating Population Variance in Two-Phase Sampling, Int. J. Sci. Eng. Res., 9 (8), 1884-1892.

7. Bhushan S. and Kumari C. (2018). Some Classes of Log Type Estimators Using Auxiliary Attribute for Population Variance, Int. J. Sci. Eng. Res., 9 (7), 1823-1832.

8. Bhushan S., Misra P. K. and Yadav S. K. (2017). On The Class of Double Sampling Exponential Ratio Type Estimator Using Auxiliary Information on an Attribute and an Auxiliary Variable, Int. J. Comp. App. Math., 12(1), 1-10.

9. Bhushan S., Gupta R. and Pandey S. K. (2015). Some log-type classes of estimators using auxiliary information, Int. J. Ag. Stat. Sci., 11(2), 487 - 491.

10. Bhushan S.(2013). Improved sampling strategies in finite population, Sch. Press, Ger- many.

11. Gupta S. and Shabbir J. (2008). Variance estimation in simple random sampling using auxiliary information, Hacet. J. Math. Stat., 37, 57-67.

12. Kadilar C. and Cingi H. (2006a). Improvement in variance estimation using auxiliary information, Hacet. J. Math. Stat., 1(35), 111-115.

13. Kadilar C. and Cingi H. (2006b). Ratio estimators for population variance in simple and stratied sampling, App. Math. Comp., 1(73), 1047-1058.

14. Kadilar C. and Cingi H. (2014). A two-parameter variance estimator using auxiliary information, App. Math. Comp., 117-122.

15. Hidiroglou M. A. and Sarndal C. E. (1998). Use of auxiliary information for two-phase sampling, Surv. Methodol., 24(1), 11-20.

16. Swain A. K. P. C. and Mishra G. (1994). Estimation of population variance under unequal probability sampling, Sankhya, B (56), 374384.

17. Bahl S. and Tuteja R. K. (1991). Ratio and Product type exponential estimator, Info. Optim. Sci., XII(I), 159-163.

18. Sukhatme P. V., Sukhatme B. V., Sukhatme S. and Ashok C. (1984) Sampling Theory of Surveys with Applications, Iowa State Univ. Press, Ames.

19. Isaki C. T. (1983). Variance estimation using Auxiliary Information, J. Amer. Stat. Asso., 78, 117-123.

20. Chaudhury A. (1978). On estimating the variance of a finite population. Metrika, 25, 66-67.

21. Das A. K. and Tripathi T. P. (1978). Use of auxiliary information in estimating the nite population variance. Sankhya, C(4), 139-148.

22. Cochran W. G. (1963). Sampling Techniques, Wiley Eastern Private Limited, New Delhi.
23. Neyman J. (1938). Contribution to the theory of sampling human populations, J. Amer. Stat. Asso., 33, 101-116.

24. Cebrian A. A. and Garcia R. M. (1997). Variance estimation using auxiliary information: an almost multivariate ratio estimator, Metrika, 45, 171-178

25. Garcia R. M. and Cebrian A. A. (1996). Repeat substitution method: the ratio estimator for population variance, Metrika, 43, 101-105.

26. Upadhaya L. N. and Singh H. P. (2001). Estimation of the population standard deviation using auxiliary information, Ame. J. Math. Manag. Sci., 21, 345-385.

27. Searls D. T. (1964). Utilization of known coefficient of kurtosis in the estimation procedure of variance, J. Amer. Stat. Assoc., 59, 1125 1226.

28. Prasad B. and Singh H. P. (1992). Unbiased estimators of finite population variance using auxiliary information sample surveys, Commun. Stat.: Theory and Methods, 21(5), 1367- 1376.

\section{AUTHOR DETAILS}

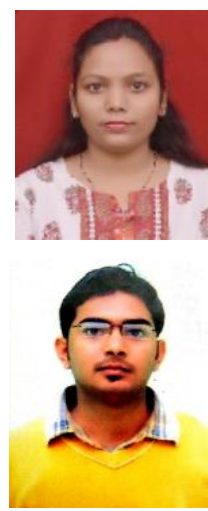

Dr. Chandni Kumari received the $\mathrm{PhD}$ degree in Statistics from Babasaheb Bhimrao Ambedkar University, Lucknow, India. Her research interests are in the areas of applied statistics. In particular, she has keen interest on Sample Surveys. She has published many research articles in reputed international and national journals.

Mr. Ratan Kumar Thakur is perusing Ph.D. in the Department of Statistics from Babasaheb Bhimrao Ambedkar University, Lucknow, India. His main area of research interests are Applied statistics, Neural Networks, Linear Regression, Time Series Forecasting, Sample Survey.

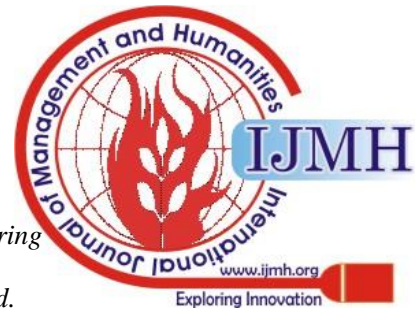

